\title{
ON THE NEGATIVE EIGENVALUES OF A CLASS OF SCHRÖDINGER OPERATORS
}

\author{
BY \\ A. LApteV AND Yu. Netrusov \\ This paper is dedicated to M.S. Birman on the occasion of \\ his 70th birthday with our warmest wishes and gratitude
}

\begin{abstract}
We find a subclass of potentials satisfying the CLR type inequalities for the number of negative eigenvalues of the operator $(-\Delta)^{l}+|x|^{-2 l}-V, l \in \mathbb{N}$, in $\mathbb{R}^{d}$ for the limiting case when $d=2 l$.
\end{abstract}

\section{InTRODUCTION}

1.1. CLR-type inequalities. We study the selfadjoint operator in $L^{2}\left(\mathbb{R}^{d}\right)$

$$
H=H_{V}=(-\Delta)^{l}+b|x|^{-2 l}-V, \quad l \in \mathbb{N}, \quad b \in \mathbb{R},
$$

where $V$ is a nonnegative, locally integrable function (potential) in $\mathbb{R}^{d}$. The operator (1.1) can be accurately defined by its quadratic form. Denote by $N_{b}(V)$ the number of negative eigenvalues of the operator (1.1).

If $2 l<d$ and $V \in L^{d / 2 l}\left(\mathbb{R}^{d}\right)$, then for any $b>-((d-2) \ldots(d-2 l))^{2} / 2^{2 l}$ the following inequality holds

$$
N_{b}(V) \leqslant C(b, d, l) \int V^{d / 2 l} d x .
$$

For $l=1(1.2)$ is known as the Cwikel-Lieb-Rozenblum (CLR) inequality.

If $2 l \geqslant d$ and $b \geqslant 0$, then the inclusion $V \in L^{d / 2 l}\left(\mathbb{R}^{d}\right)$ does not imply (1.2). In fact, this inclusion does not guarantee even the semiboundedness of the operator (1.1) from below. For $2 l>d$ some different type estimates of the number of the negative eigenvalues were obtained in [BS1] for $d$ odd and in [BLS] for $d$ even. In the case $2 l=d$ the corresponding results are less complete (see [BL], [BLS], $[\mathrm{L}]$ and $[\mathrm{S} 1,2])$. It was first shown in $[\mathrm{S} 1,2]$, and then in a sharper form in [BL] and [BLS], that if $2 l=d$ and $b=0$, then the problem can be separated into two problems. The first one is defined by the restriction of the operator (1.1) to the subspace of functions depending on $|x|$ and, hence, is reduced to a well studied one-dimensional

1991 Mathematics Subject Classification. 35P15, 35P20.

The first author was supported by the Swedish Natural Sciences Research Council, Grant MAA/MA 09364-320. The second author was supported by the British Engineering and Physical Sciences Research Council, Grant AF/97/1657 and by the Royal Swedish Academy of Science (project number 1400). 
differential operator with the potential equal to the mean value $\widetilde{V}$ of $V$ over $\mathbb{S}^{d-1}$. In particular, for this class of operators there are (see [BS1]) necessary and sufficient conditions on the potential $\widetilde{V}$ which give $N_{0}(\alpha \widetilde{V})=O(\alpha)$, as $\alpha \rightarrow \infty$. The second problem is defined by a class of functions whose mean values over $\mathbb{S}^{d-1}$ are equal to zero. On this subspace we have the Hardy inequality which automatically provides us with the "supporting" term $b|x|^{-2 l}$ with some $b>0$. This suggests that in order to study the case $2 l=d$, we have to pay special attention to the operator (1.1), where $b>0$.

The purpose of this paper is to find a subclass of potentials from $L^{1}\left(\mathbb{R}^{d}\right)$, such that the inequality (1.2) holds for $d=2 l$ and $b>0$. We shall always assume that $b=1$. For an arbitrary $b>0$ all the statements of this paper remain true but the constants depend on $b$. For $b=1$ (1.2) takes the form

$$
N(V)=N_{1}(V) \leqslant C(d) \int V(x) d x
$$

The right hand side of (1.3) does not require more than $V \in L^{1}\left(\mathbb{R}^{d}\right)$. We prefer to deal with the problem

$$
H_{\mu}=(-\Delta)^{l}+|x|^{-2 l}-\mu,
$$

where $\mu$ is a nonnegative, finite measure in $\mathbb{R}^{d}$. If $\mu$ is an absolutely continuous measure, $d \mu=V d x$, and $b=1$, then (1.4) coincides with (1.1). Let $\left|\nabla^{l} u\right|^{2}:=$ $\sum_{|\beta|=l}(l ! / \beta !)\left|\partial^{\beta} u\right|^{2}$. We shall impose such conditions on $\mu$ that the quadratic form

$$
h_{\mu}[u, u]=\int\left(\left|\nabla^{l} u\right|^{2}+|x|^{-2 l}|u|^{2}\right) d x-\int|u|^{2} d \mu
$$

defined on $\mathcal{H}^{l}\left(\mathbb{R}^{d}\right)$ (see(1.8)) is semibounded and closed in $L^{2}\left(\mathbb{R}^{d}\right)$ and, hence, defines a selfadjoint operator $H_{\mu}$. Notice that necessary and sufficient conditions of closability and semiboundedness of a wide classes of quadratic forms were obtained in $[\mathrm{M}, \mathrm{Ch} .8$ and 12$]$.

For $b=0$ the operator (1.1) has already been studied in [S1,2], where some estimates of $N_{0}(V)$ were obtained in terms of Orlicz classes. This paper deals with the problem of finding a class of potentials, such that the prescribed inequality (1.3) is satisfied. Our conditions are different, and the results of this paper and those obtained in [S1,2] complement each other. In particular, if $d \mu=V d x$ and $V(x)=V(|x|) \in L^{1}\left(\mathbb{R}^{d}\right), d=2 l$, then our results imply the inequality (1.3) (see also $[\mathrm{L}])$.

1.2. The main results. In order to formulate the main result we introduce the following definition and notation.

The open ball with centre at $x \in \mathbb{R}^{d}$ and radius $r>0$ is denoted by $B(x, r)$,

$$
B(x, r)=\left\{y \in \mathbb{R}^{d}:|y-x|<r\right\} .
$$

Condition $(*)$. Let $\mu$ be a nonnegative measure in $\mathbb{R}^{d}, d \geqslant 1$, whose support $F=\operatorname{supp} \mu$ is a bounded set. We say that the measure $\mu$ satisfies Condition $(*)$ with constants $\gamma_{1}$ and $\gamma_{2}$, where $\gamma_{1}, \gamma_{2}>1$, if for any $x \in \mathbb{R}^{d}$ and $r \leqslant \gamma_{2}^{-1} \operatorname{diam} F$ we have that 
Denote

$$
\begin{gathered}
\Omega_{c_{1}, c_{2}}:=\left\{x \in \mathbb{R}^{d}: c_{1} \leqslant|x|<c_{2}\right\}=B\left(0, c_{2}\right) \backslash B\left(0, c_{1}\right), \quad 0 \leqslant c_{1}<c_{2} \leqslant \infty, \\
\Omega_{k}:=\Omega_{c_{1}, c_{2}}, \quad \text { where } \quad c_{1}=2^{k-1}, \quad c_{2}=2^{k}, \quad k \in \mathbb{Z}
\end{gathered}
$$

and

$$
\widetilde{\Omega}_{k}:=\Omega_{c_{1}, c_{2}}, \quad \text { where } \quad c_{1}=2^{k-2}, \quad c_{2}=2^{k+1}, \quad k \in \mathbb{Z} .
$$

Our main result is the following statement:

Theorem 1.1. Let $\mu$ be a finite, nonnegative Borel measure in $\mathbb{R}^{d}$, $d$ even, whose restrictions $\left.\mu\right|_{\bar{\Omega}_{k}}, k \in \mathbb{Z}$ satisfy Condition (*) with constants $\gamma_{1}$ and $\gamma_{2}$ independent of $k$. Then the quadratic form (1.5) is semibounded from below, closed on $\mathcal{H}^{l}\left(\mathbb{R}^{d}\right)$ and the number of negative eigenvalues $N(\mu)$ of the corresponding operator

$$
H_{\mu}=(-\Delta)^{l}+|x|^{-2 l}-\mu, \quad l=d / 2 \in \mathbb{N},
$$

satisfies

$$
N(\mu) \leqslant C \mu\left(\mathbb{R}^{d}\right)
$$

where $C=C\left(\gamma_{1}, \gamma_{2}, d\right)$.

The proof of this theorem of is given in Section 4 .

Remark 1.1. It can be easily checked (see Section 5) that Condition (*) is satisfied for any spherically symmetric measure. In particular, if $\mu$ is the $\delta$-function of $\mathbb{S}^{d-1}$, then the constants $\gamma_{1}$ and $\gamma_{2}$ can for example, be chosen as $\gamma_{1}=\gamma_{2}=2$. In fact, Condition $(*)$ is satisfied for the $\delta$-function of an arbitrary compact smooth submanifold of $\mathbb{R}^{d}$ of a positive dimension.

The next result is related to absolutely continuous measures $\mu=V d x$. Its proof follows from Theorem 1.1, but requires some additional technical preparations (see Section 5). Let us introduce a class of functions $L^{1}\left(\mathbb{R}_{+}, L^{p}\left(\mathbb{S}^{d-1}\right)\right)$ defined in polar coordinates in $\mathbb{R}^{d}, x=(r, \theta), r \in \mathbb{R}_{+}=(0, \infty)$, as

$$
\|f\|_{L^{1}\left(\mathbb{R}_{+}, L^{p}\left(\mathbb{S}^{d-1}\right)\right)}=\int_{0}^{\infty}\left(\int_{\mathbb{S}^{d-1}}|f(r, \theta)|^{p} d \theta\right)^{1 / p} r^{d-1} d r<\infty .
$$

Theorem 1.2. Let $d$ be even, $l=d / 2, V \geqslant 0$ and $V \in L^{1}\left(\mathbb{R}_{+}, L^{p}\left(\mathbb{S}^{d-1}\right)\right), 1<p \leqslant$ $\infty$. Then the number of the negative eigenvalues of the operator (1.1) with $b=1$ satisfies

$$
N(V) \leqslant C\|V\|_{L^{1}\left(\mathbb{R}_{+}, L^{p}\left(\mathbb{S}^{d-1}\right)\right)}
$$

where $C=C(d, p)$.

In particular, we immediately obtain the following

Corollary 1.3. Let $l=d / 2 \in \mathbb{N}, V \geqslant 0$ and $V(x)=V(|x|)$. If $V \in L^{1}\left(\mathbb{R}^{d}\right)$, then the inequality (1.3) is fulfilled.

Remark 1.2. Even for the class of spherically symmetric potentials the inequality (1.3) fails if we do not introduce the "supporting" term $|x|^{-2 l}$. Indeed, as it was shown in [BL] and in [BLS], if $\mu=V(x) d x$ and $V$ is a smooth potential, such that

$$
V(x) \sim|x|^{-2 l} \ln ^{-2}|x|(\ln \ln |x|)^{-1 / q}, \quad \text { as } \quad|x| \rightarrow \infty, \quad q>1,
$$

then the number of negative eigenvalues of the operator $(-\Delta)^{l}-V$ satisfies the following asymptotic formula

$$
N_{0}(\alpha V)=\alpha^{q} c_{q}+o\left(\alpha^{q}\right), \quad \alpha \rightarrow \infty
$$


1.3. Some notation. We shall denote by $L^{p}\left(\mathbb{R}^{d}, \mu\right)$ the class of $L^{p}$-integrable functions with respect to a measure $\mu$. If $\mu$ coincides with the Lebesgue measure, then we omit $\mu$ and write $L^{p}\left(\mathbb{R}^{d}, \mu\right)=L^{p}\left(\mathbb{R}^{d}\right)$. Let $G$ be an open subset of $R^{d}$. By $H^{l}(G)$ we denote the Sobolev class of the order $l$ equipped by the standard Hilbert metric

$$
H^{l}(G)=\left\{f: \int_{G}\left(\left|\nabla^{l} f\right|^{2}+|f|^{2}\right) d x<\infty\right\} .
$$

The integral over the whole space is written without indicating the domain of integration. The class of functions $\mathcal{H}^{l}\left(\mathbb{R}^{d}\right), l=d / 2, l \in \mathbb{N}$, is a so-called homogeneous $H^{l}$ class and defined by

$$
\mathcal{H}^{l}\left(\mathbb{R}^{d}\right)=\left\{f: \int\left(\left|\nabla^{l} f\right|^{2}+\frac{|f|^{2}}{|x|^{2 l}}\right) d x<\infty\right\} .
$$

$C$ and $c$ will be different constants whose values are unimportant. By $\mathcal{P}^{l}$ is denoted the class of polynomials in $\mathbb{R}^{d}$ of degree less than or equal to $l$. By $v_{d}$ we denote the volume of the unit ball in $\mathbb{R}^{d}$,

$$
v_{d}:=|B(0,1)|=\operatorname{vol}\left\{x \in \mathbb{R}^{d}:|x|<1\right\}=\frac{\pi^{d / 2}}{\Gamma(1+d / 2)} .
$$

Acknowledgments. The authors are grateful to L.I. Hedberg, V.G. Maz'ya and M. Solomyak for useful remarks and discussions.

\section{Covering Lemmas}

Let us first recall a classical result of Besicovitch [B1,2] (see also [G], Ch.1)

Lemma 2.1. Let $A \subset \mathbb{R}^{d}, d \geqslant 1$, be a compact set and $r$ be a positive function on $A$. Then there exists a finite subset $J \subset A$ and a family of balls $\{B(x, r(x))\}_{x \in J}$, such that the following two conditions are fulfilled:

(1) $\cup_{x \in J} B(x, r(x)) \supset A$,

(2) for any $y \in A$

$$
\#\{x: x \in J \text { and } \bar{B}(x, r(x)) \ni y\} \leqslant C,
$$

where the constant $C=C(d)$ depends only on the dimension $d$.

Lemma 2.1 was first applied for the problem of spectral estimates in [BS2]. The next result follows from Lemma 2.1 and already appeared in $[R]$ for absolutely continuous measures. It will be used in the proof of Theorem 1.1.

Lemma 2.2. Let $\mu$ be a finite, continuous, nonnegative Borel measure in $\mathbb{R}^{d}$. Suppose that its support $F=\operatorname{supp} \mu$ is a bounded set. Then for any $m \in \mathbb{N}$ there exists a finite set $J \subset F$ and a family of balls $\{B(x, r(x))\}_{x \in J}$ satisfying the following conditions:

(1) $\cup_{x \in J} B(x, r(x)) \supset F$,

(2) $\mu(B(x, r(x))) \leqslant \frac{\mu\left(\mathbb{R}^{d}\right)}{m} \leqslant \mu(\bar{B}(x, r(x))), \quad x \in J$,

(3) for any $y \in \mathbb{R}^{d}$

$$
\#\{x: x \in J \text { and } y \in \bar{B}(x, r(x))\} \leqslant C_{1}(d),
$$

(4) $\#\{x: x \in J\} \leqslant C_{2}(d) m$. Here the constants $C_{1}$ and $C_{2}$ depend only on 
Proof. For an arbitrary $x \in F$ and $m \in \mathbb{N}$ define

$$
\widetilde{r}(x)=\sup \left\{r(x) \in \mathbb{R}_{+}: \mu\left(B(x, r(x)) \leqslant \frac{\mu\left(\mathbb{R}^{d}\right)}{m}\right\}\right.
$$

Then

and obviously

$$
\mu(B(x, \widetilde{r}(x))) \leqslant \frac{\mu\left(\mathbb{R}^{d}\right)}{m}, \quad x \in F
$$

$$
\mu(\bar{B}(x, \widetilde{r}(x))) \geqslant \frac{\mu\left(\mathbb{R}^{d}\right)}{m}, \quad x \in F .
$$

Applying now Lemma 2.1 we find a finite set $J$ such that (1)-(3) are fulfilled. Since the support of $F$ is a compact set, then (3) and (2.2) imply (4). The proof is complete.

Remark 2.1. If supp $\mu \subset \bar{\Omega}_{1}=\overline{B(0,2)} \backslash B(0,1)$, then in Lemma 2.2 we could choose the family of covering balls $\{B(x, r(x))\}, x \in J$, such that their supports were lying in $\operatorname{Int} \widetilde{\Omega}_{1}=\operatorname{Int}(B(0,4) \backslash B(0,1 / 2))$. Indeed, when introducing $\widetilde{r}(x)$, we could require in addition that the supremum is taken over $r(x) \leqslant 1 / 2$. Then the proof of the conditions (1)-(3) remains the same. The estimate for the number of points $x \in J$ satisfying (4) with $\widetilde{r}(x)<1 / 2$ is the same, but the number of balls with $\widetilde{r}(x)=1 / 2$ is bounded.

\section{Some inequalities From REAL ANAlysis}

We collect here preliminary material which prevents us from being distracted while proving the main result.

The next statement is a version of the well-known Poincaré inequality (see, for example, [M, Ch.1.1.11] or [AH, Ch.8.1]).

Lemma 3.1. For any $l \in \mathbb{N}$ and any ball $B(0, r) \subset \mathbb{R}^{d}, r>0$, there exists a linear operator (orthogonal projection in $L^{2}(B(0, r)$ )

$$
T: L^{2}(B(0, r)) \hookrightarrow \mathcal{P}^{l-1}
$$

such that

$$
\|f-T f\|_{L^{2}(B(0, r))}^{2} \leqslant C(d) r^{2 l}\left\|\nabla^{l} f\right\|_{L^{2}(B(0, r))}^{2} .
$$

The proof of the following statement is due to Adams [A] (see also [M, Ch.8] and $[\mathrm{AH}, \mathrm{Th} .7 .2 .2]$ where there are also many other related results). It concerns Sobolev spaces $H^{\alpha}$ of any (not necessarily integer) positive order $\alpha$.

Theorem 3.2. Let $\alpha<d / 2$ and $\mu$ be a finite, nonnegative measure in $\mathbb{R}^{d}, F=$ supp $\mu \subset B(0,1)$ and suppose that there is a constant $C_{*}$, such that for some $\beta>0$ and any $r, 0<r<\infty$

$$
\mu(B(x, r)) \leqslant C_{*} r^{\beta}, \quad x \in F .
$$

If $\frac{\beta}{p}=\frac{d}{2}-\alpha, p>2$, then the embedding operator

$$
H^{\alpha}(B(0,1)) \hookrightarrow L^{p}(B(0,1), \mu)
$$

is bounded and its norm does not exceed $c C_{*}^{1 / p}$, where $c=c(\alpha, d, \beta)$ is independent of the measure $\mu$.

In particular, this theorem implies the following weaker result for the case $2 \alpha=$ 
Corollary 3.3. Let $l=d / 2$ and $\mu$ be a finite, non-negative measure in $\mathbb{R}^{d}, F=$ $\operatorname{supp} \mu \subset B(0,1)$ and suppose there exists a constant $C_{*}$, such that for some $\beta>0$ and any $r, 0<r<\infty$,

$$
\mu(B(x, r)) \leqslant C_{*} r^{\beta}, \quad x \in F .
$$

Then the embedding operator

$$
H^{l}(B(0,1)) \hookrightarrow L^{2}(B(0,1), \mu)
$$

is bounded and its norm does not exceed $C C_{*}^{1 / 2}$, where $C=C(l, \beta)$.

Proof. The proof is very simple. Choose $p>1$ and $\alpha^{\prime}$, such that $l>\alpha^{\prime}=d / 2-$ $\beta / 2 p>0$. Applying the Hölder inequality with $q=p /(p-1)$ and Theorem 3.2 we find

$$
\begin{array}{r}
\|u\|_{L^{2}(B(0,1), \mu)} \leqslant\|u\|_{L^{2 p}(B(0,1), \mu)} \mu^{1 / 2 q}(B(0,1)) \leqslant c C_{*}^{1 / 2 p}\|u\|_{H^{\alpha^{\prime}}(B(0,1))} C_{*}^{1 / 2 q} \\
\leqslant C C_{*}^{1 / 2}\|u\|_{H^{l}(B(0,1))} .
\end{array}
$$

This completes the proof.

Using dilation and Corollary 3.3 we obtain (see [M, Lemma 1.4.7])

Lemma 3.4. Let $l=d / 2 \in \mathbb{N}, \beta>0, \mu$ nonnegative, finite measure in $\mathbb{R}^{d}$ and supp $\mu \subset \bar{B}(0, r), r>0$. Then there exists a constant $C=C(\beta, d)$, such that

$$
\|f\|_{L^{2}(B(0, r), \mu)}^{2} \leqslant C r^{\beta} M\left(\left\|\nabla^{l} f\right\|_{L^{2}(B(0, r))}^{2}+r^{-d}\|f\|_{L^{2}(B(0, r))}^{2}\right),
$$

where

$$
M=\sup _{x \in \mathbb{R}^{d}, \rho>0} \frac{\mu(B(x, \rho))}{\rho^{\beta}}
$$

and $C=C(l, \beta)$.

Lemmas 3.1 and 3.4 immediately give us

Corollary 3.5. Let $T$ be the orthogonal projection defined in (3.1), $l=d / 2 \in \mathbb{N}$, $\beta>0$, let $\mu$ be a nonnegative, finite measure in $\mathbb{R}^{d}$ and $\operatorname{supp} \mu \subset \bar{B}(0, r), r>0$. Then there exists a constant $C=C(\beta, d)$, such that

$$
\|f-T f\|_{L^{2}(B(0, r), \mu)}^{2} \leqslant C r^{\beta} M\left\|\nabla^{l} f\right\|_{L^{2}(B(0, r))}^{2},
$$

where $M$ is given by (3.3) and $C=C(l, \beta)$.

Remark 3.1. When proving the next lemma we use the following simple remark: if $\mu$ satisfies Condition $(*)$ with the constants $\left(\gamma_{1}, \gamma_{2}\right)$, then there are constants $\alpha>0$ and $\varkappa>0$ such that for any $0<r<\infty, \gamma>1$ and $\gamma r \leqslant \gamma_{1} \operatorname{diam} F / \gamma_{2}$ we have

$$
\mu(B(x, \gamma r)) \geqslant \varkappa \gamma^{\alpha} \mu(B(x, r)), \quad x \in \mathbb{R}^{d} .
$$


Let $m, L \in \mathbb{N}, A>1$ be constants satisfying the inequalities $A^{L} / m \leqslant 1<$ $A^{L+1} / m$ and let $C_{1}(d)$ be the constant appearing in Lemma 2.1. By using Lemma 2.2 we choose a family of balls $B_{v, j}=B\left(x_{v j}, r_{v j}\right), x_{v j} \in \mathbb{R}^{d}, r_{v j}>0, v=0,1, \ldots, L$, $j=1,2, \ldots, n_{v}$, so that for any $v=0,1, \ldots, L$ the following conditions are fulfilled:

$$
\begin{gathered}
n_{v} \leqslant C(A, d) m A^{-v}, \\
F \subset \cup_{j=1}^{n_{v}} B_{v, j}, \quad \#\left\{j: 1 \leqslant j \leqslant n_{v}, y \in \bar{B}_{v, j}\right\} \leqslant C_{1}(d)
\end{gathered}
$$

for any $y \in \mathbb{R}^{d}$ and

$$
\mu\left(B_{v, j}\right) \leqslant \frac{A^{v} \mu\left(\mathbb{R}^{d}\right)}{m} \leqslant \mu\left(\bar{B}_{v, j}\right), \quad j=1,2, \ldots, n_{v}
$$

Let $\Lambda=\Lambda(d)$ denote the maximum number of balls with the following properties: i) radii of the balls do not exceed $1 / 2$; ii) all the balls intersect $B(0,1)$; iii) any point $x \in \mathbb{R}^{d}$ belongs to not more than $C_{1}(d)$ balls.

Lemma 3.6. Let $\mu$ be a finite, nonnegative measure in $R^{d}, F=\operatorname{supp} \mu$ be a bounded set and $\mu$ satisfies Condition $(*)$ with the constants $\left(\gamma_{1}, \gamma_{2}\right)$. Let $A, m$, $L, \Lambda(d)$ be the constants and $\left\{B_{v, j}\right\}_{j=1}^{n_{v}}, v=0,1, \ldots, L$, be the families of balls introduced above. Then for any ball $B(x, r)$ satisfying

$$
\mu(B(x, r)) \geqslant \frac{K \mu\left(\mathbb{R}^{d}\right)}{m},
$$

there exists a ball $B_{u, i}=B\left(x_{u i}, r_{u i}\right), 0 \leqslant u \leqslant L-1,1 \leqslant i \leqslant n_{u}$, with the properties

$$
\left|x-x_{u i}\right| \leqslant 3 r \quad \text { and } \quad \frac{r}{2^{u}} \geqslant r_{u i} \geqslant \frac{r}{\zeta 2^{u}}
$$

where $\zeta$ is defined by $(\zeta / 2)^{\alpha} \varkappa=K A$ and $K>\max (\Lambda(d), A)$.

Proof. From the assumptions

$$
K>\Lambda(d), \quad \mu(B(x, r)) \geqslant \frac{K \mu\left(\mathbb{R}^{d}\right)}{m}, \quad \mu\left(B_{0, j}\right) \leqslant \frac{\mu\left(\mathbb{R}^{d}\right)}{m}, \quad j=1,2, \ldots, n_{0},
$$

it follows that there exists $j_{0}$ such that $B_{0, j_{0}} \cap B(x, r) \neq \varnothing$ and $r_{0 j_{0}}<r / 2$. If $r_{0 j_{0}} \geqslant r / \zeta$, then the statement of the lemma is fulfilled if we take $B_{u, i}=B_{0, j_{0}}$. Thus we can assume that $r_{0 j_{0}}<r / \zeta$. Let us introduce a new ball $B_{1}=B\left(x_{1}, r / 2\right)=$ $B\left(x_{0 j_{0}}, r / 2\right)$. Then (3.5) implies

$$
\mu\left(B_{1}\right) \geqslant \varkappa\left(\frac{r / 2}{r / \zeta}\right)^{\alpha} \mu\left(\bar{B}_{0, j_{0}}\right)>\varkappa\left(\frac{\zeta}{2}\right)^{\alpha} \frac{\mu\left(\mathbb{R}^{d}\right)}{m}=\frac{K A \mu\left(\mathbb{R}^{d}\right)}{m}
$$

and

$$
\left|x_{1}-x\right| \leqslant r+r / 2 .
$$

At the next step we repeat our arguments for the ball $B\left(x_{1}, r / 2\right)$ instead of $B(x, r)$ and the family $\left\{B_{1, j}\right\}_{j=1}^{n_{1}}$ instead of $\left\{B_{0, j}\right\}_{j=1}^{n_{0}}$. By using the inequalities

$$
K>\Lambda(d) \quad \mu\left(B\left(x_{1}, r / 2\right)\right) \geq \frac{A K \mu\left(\mathbb{R}^{d}\right)}{}
$$




$$
\mu\left(B_{1, j}\right) \leqslant \frac{A \mu\left(\mathbb{R}^{d}\right)}{m}, \quad j=1,2, \ldots, n_{1},
$$

we find $j_{1}$ such that $B\left(x_{1}, r / 2\right) \cap B\left(x_{1 j_{1}}, r_{1 j_{1}}\right) \neq \varnothing$ and $r_{1 j_{1}}<r / 4$. If $r_{1 j_{1}}>r / 2 \zeta$, then the statement of the lemma is fulfilled if we take $B_{u, i}=B_{1, j_{1}}$. Thus we can assume that $r_{1 j_{1}}<r / 2 \zeta$ and introduce $B_{2}=B\left(x_{2}, r / 4\right)=B\left(x_{1 j_{1}}, r / 4\right)$. Then by again applying (3.5) we obtain

$$
\mu\left(B_{2}\right) \geqslant \varkappa\left(\frac{\zeta}{2}\right)^{\alpha} \frac{A \mu\left(\mathbb{R}^{d}\right)}{m} \geqslant \frac{K A^{2} \mu\left(\mathbb{R}^{d}\right)}{m}
$$

and

$$
\left|x_{1}-x\right| \leqslant\left|x_{1}-x\right|+\left|x_{2}-x_{1}\right| \leqslant r+r / 2+r / 2+r / 4 .
$$

Continuing this process we either find a ball $B_{u, i}, 0 \leqslant u \leqslant L-1,1 \leqslant i \leqslant n_{u}$, satisfying the statement of the lemma or arrive at a ball $B_{L}$ with the property

$$
\mu\left(B_{L}\right) \geqslant \frac{K A^{L}}{m} \mu\left(\mathbb{R}^{d}\right)>\frac{A^{L+1}}{m} \mu\left(\mathbb{R}^{d}\right)
$$

The last inequality is impossible since $A^{L+1} / m>1$ and, therefore, the proof is complete.

Let $x_{0} \in \mathbb{R}^{d}, 0<r_{0}<\infty$ and $0<\beta<\alpha$. Denote

$$
\varphi\left(B\left(x_{0}, r_{0}\right)\right)=r_{0}^{\beta} \sup _{x \in B\left(x_{0}, r_{0}\right), 0<r<r_{0}} r^{-\beta} \mu\left(B(x, r) \cap B\left(x_{0}, r_{0}\right)\right) .
$$

Correspondingly the value $\varphi\left(\bar{B}\left(x_{0}, r_{0}\right)\right)$ is defined by $(3.7)$, where the open ball $B(x, r)$ is changed by $\bar{B}(x, r)$.

Lemma 3.7. Let $l=d / 2 \in \mathbb{N}, \mu$ be a nonnegative, finite measure satisfying Condition $(*)$ with the constants $\left(\gamma_{1}, \gamma_{2}\right)$ and supp $\mu \subset \bar{\Omega}_{1}$. Then for any $m \in \mathbb{N}$ there exists a subspace $E \subset H^{l}\left(\widetilde{\Omega}_{1}\right)$, such that $\operatorname{dim} E \leqslant C \cdot m$ and for any $f \in H^{l}\left(\widetilde{\Omega}_{1}\right)$, $f \perp E$ we have

$$
\int_{\bar{\Omega}_{1}}|f(x)|^{2} d \mu(x) \leqslant C^{\prime} \frac{\mu\left(\bar{\Omega}_{1}\right)}{m} \int_{\widetilde{\Omega}_{1}}\left|\nabla^{l} f(x)\right|^{2} d x,
$$

where $C^{\prime}=C^{\prime}\left(d, \gamma_{1}, \gamma_{2}\right), C=C\left(d, \gamma_{1}, \gamma_{2}\right)$.

Proof. Let us assume that we can find a family of balls $\left\{B_{k}\right\}_{k=1}^{S}$ satisfying the properties:

$$
S \leqslant C_{0} m,
$$

$$
F \subset \cup_{k=1}^{S} B_{k},
$$


for any $y \in \mathbb{R}^{d}$ and

$$
\varphi\left(B_{k}\right) \leqslant \frac{\mu\left(\mathbb{R}^{d}\right)}{m} \leqslant \varphi\left(\bar{B}_{k}\right), \quad k=1, \ldots, S
$$

Denote by $E$ the orthogonal complement of the subspace $H^{l}\left(\widetilde{\Omega}_{1}\right)$ defined by $\int_{B_{k}} f p d x=0, k=1, \ldots, S$, where $p \in \mathcal{P}^{l-1}$, (see Lemma 3.1). It follows from Corollary 3.5 and (3.11) that for any function $f \perp E$

$$
\int_{B_{k}}|f|^{2} d \mu \leqslant C_{2} \frac{\mu\left(\mathbb{R}^{d}\right)}{m} \int_{B_{k}}\left|\nabla^{l} f\right|^{2} d x
$$

Then using the last inequality, (3.9) and (3.10) we obtain the required statement.

Therefore in order to finish the proof of the lemma we need to construct a family of balls satisfying conditions (3.8)-(3.11).

From (3.5) and (3.7) it is easy to see that $\lim _{r \rightarrow 0} \varphi(B(x, r))=0$. By applying Lemma 2.2 , where $\varphi$ is used instead of $\mu$, we find a family of balls $\left\{B_{k}\right\}_{k=1}^{S}$ such that (3.9)-(3.11) are fulfilled. We only need to check (3.8).

Choose $0<\delta \leqslant(\varkappa / K)^{\frac{\beta}{\beta-\alpha}}$. Let us split the family $\left\{B_{k}\right\}_{k=1}^{S}$ into two sets of balls which after renumbering satisfy

$$
\mu\left(\bar{B}_{k}\right)<\frac{\delta \mu\left(\mathbb{R}^{d}\right)}{m}, \quad 1 \leqslant k \leqslant s
$$

and

$$
\mu\left(\bar{B}_{k}\right) \geqslant \frac{\delta \mu\left(\mathbb{R}^{d}\right)}{m}, \quad s+1 \leqslant k \leqslant S
$$

The condition (3.10) gives us $S-s \leqslant C_{1}(d) / \delta m$. Thus in order to complete the proof of (3.8) it is enough to verify the estimate

$$
s \leqslant C_{3} m .
$$

From now on we use the notations from Lemma 3.6. Let us claim that for any $B_{k}=B\left(x_{k}, r_{k}\right), 1 \leqslant k \leqslant s$, there is a ball $B_{u_{k}, i_{k}}=B\left(x_{u_{k} i_{k}}, r_{u_{k} i_{k}}\right)$ with the properties

$$
\left|x_{k}-x_{u_{k} i_{k}}\right| \leqslant 4 r_{k}, \quad r_{k} / \zeta 2^{u} \leqslant r_{u_{k} i_{k}} \leqslant r_{k} / 2^{u}
$$

Then from these inequalities and (3.10) we find that for any $0 \leqslant v \leqslant L-1$ and $1 \leqslant j \leqslant n_{v}$

$$
\#\left\{k: 1 \leqslant k \leqslant s, u_{k}=v, i_{k}=j\right\} \leqslant C_{4}(d) C_{1}(d)=C_{5} .
$$

Hence by (3.6)

$$
s \leqslant C_{5}\left(n_{0}+n_{1}+\cdots+n_{L-1}\right) \leqslant C_{5} C(A, d) m \sum_{v=0}^{L-1} A^{-v} \leqslant C_{6}(A, d) m
$$


Let us prove our claim. From (3.11) we conclude that for any $1 \leqslant k \leqslant s$ there exists a ball $B\left(y_{k}, \rho_{k}\right)$, such that $\rho_{k} \leqslant r_{k}, y_{k} \in \bar{B}_{k}$ and

$$
\mu\left(\bar{B}\left(y_{k}, \rho_{k}\right) \cap \bar{B}\left(x_{k}, r_{k}\right)\right)\left(r_{k} / \rho_{k}\right)^{\beta} \geqslant \frac{\mu\left(\mathbb{R}^{d}\right)}{m} .
$$

The latter and (3.12) imply

$$
\left(\frac{r_{k}}{\rho_{k}}\right)^{\beta} \delta>1
$$

Using now (3.5), (3.14) and (3.15) we obtain

$$
\begin{aligned}
& \mu\left(B\left(y_{k}, r_{k}\right)\right) \geqslant \varkappa\left(\frac{r_{k}}{\rho_{k}}\right)^{\alpha} \mu\left(\bar{B}\left(y_{k}, \rho_{k}\right)\right) \\
& \geqslant \varkappa\left(\frac{r_{k}}{\rho_{k}}\right)^{\alpha} \mu\left(\bar{B}\left(y_{k}, \rho_{k}\right) \cap \bar{B}\left(x_{k}, r_{k}\right)\right) \\
& \geqslant \varkappa\left(\frac{r_{k}}{\rho_{k}}\right)^{\alpha-\beta} \frac{\mu\left(\mathbb{R}^{d}\right)}{m} \\
& \geqslant \varkappa\left(\frac{1}{\delta^{1 / \beta}}\right)^{\alpha-\beta} \frac{\mu\left(\mathbb{R}^{d}\right)}{m} \geqslant \frac{K \mu\left(\mathbb{R}^{d}\right)}{m},
\end{aligned}
$$

where the last inequality follows from the choice of the constant $\delta$. By applying Lemma 3.6 to $B\left(y_{k}, r_{k}\right)$ we find the required ball $B_{u_{k}, i_{k}}$ and hence prove the claim and the lemma.

From Lemma 3.4 and Condition $(*)$ also we obtain the following statement:

Lemma 3.8. Let $l=d / 2 \in \mathbb{N}$ and let $\mu$ be a nonnegative, finite measure satisfying Condition $(*)$ with the constants $\left(\gamma_{1}, \gamma_{2}\right)$ and $\operatorname{supp} \mu \subset \bar{\Omega}_{1}$. Then

$$
\int_{\bar{\Omega}_{1}}|f(x)|^{2} d \mu(x) \leqslant C^{\prime \prime} \mu\left(\bar{\Omega}_{1}\right)\left(\int_{\widetilde{\Omega}_{1}}\left|\nabla^{l} f(x)\right|^{2} d x+\int_{\widetilde{\Omega}_{1}}|f(x)|^{2} d x\right),
$$

where $C^{\prime \prime}=C\left(d, \gamma_{1}, \gamma_{2}\right)$.

\section{Proof of Theorem 1.1}

According to the variational principle, in order to prove Theorem 1.1 it is sufficient to show that there exists a subspace $E_{0} \subset H^{l}\left(\mathbb{R}^{d}\right), \operatorname{dim} E_{0} \leqslant C \mu\left(\mathbb{R}^{d}\right)$, such that for any $F \in \mathcal{H}^{l}$ and $f \perp E_{0}$ in $L^{2}\left(\mathbb{R}^{d}\right)$ we have the following inequality

$$
\int_{\mathbb{R}^{d}}|f|^{2} d \mu \leqslant \int_{R^{d}}\left|\nabla^{l} f\right|^{2} d x+\int_{R^{d}} \frac{|f|^{2}}{|x|^{2 l}} d x
$$

Let us denote by $\mu_{k}$ the restriction of $\mu$ on the set $\Omega_{k}$. Introduce

$$
\mathcal{K}:=\left\{k:\left\|\mu_{k}\right\|_{1}>\frac{1}{3 \cdot 2^{d} \cdot C^{\prime \prime}}\right\}
$$


Lemma 3.7 implies that for any $k \in \mathcal{K}$ and any $m=m_{k} \in \mathbb{N}$ we can find a $C(d) \cdot m_{k}$ - dimensional subspace $E_{k} \subset L^{2}\left(\widetilde{\Omega}_{k}\right)$, such that for $f \perp E_{k}$ we have

$$
\begin{aligned}
& \int_{\bar{\Omega}_{k}}|f(x)|^{2} d \mu(x)=\int_{\bar{\Omega}_{1}}\left|f\left(2^{k} x\right)\right|^{2} d \mu\left(2^{k} x\right) \\
& \leqslant C^{\prime} \frac{\mu\left(2^{k} \Omega_{1}\right)}{m_{k}} \int_{\widetilde{\Omega}_{1}}\left|\nabla^{l} f\left(2^{k} x\right)\right|^{2} d x \leqslant C^{\prime} \frac{\mu\left(\Omega_{k}\right)}{m_{k}} \int_{\widetilde{\Omega}_{k}}\left|\nabla^{l} f(x)\right|^{2} d x
\end{aligned}
$$

Notice that if we now choose $m_{k}=3\left[\left(1+C^{\prime}\right) \cdot \mu\left(\Omega_{k}\right)\right]$, then

$$
\int_{\bar{\Omega}_{k}}|f(x)|^{2} d \mu(x) \leqslant \frac{1}{3} \int_{\widetilde{\Omega}_{k}}\left|\nabla^{l} f(x)\right|^{2} d x,
$$

and moreover

$$
\sum_{k \in \mathcal{K}} m_{k} \leqslant 3\left(1+C^{\prime}\right) \cdot \mu\left(\mathbb{R}^{d}\right)
$$

Assume now that $k \notin \mathcal{K}$. Then Lemma 3.8 and the definition of the set $\mathcal{K}$ give us

$$
\begin{aligned}
& \int_{\bar{\Omega}_{k}}|f(x)|^{2} d \mu(x)= \int_{\bar{\Omega}_{1}}\left|f\left(2^{k} x\right)\right|^{2} d \mu\left(2^{k} x\right) \\
& \leqslant C^{\prime \prime} \mu\left(2^{k} \Omega_{1}\right)\left.\left.\left(\int_{\widetilde{\Omega}_{1}} \mid \nabla^{l} f\left(2^{k} x\right)\right)\right|^{2} d x+\int_{\widetilde{\Omega}_{1}}\left|f\left(2^{k} x\right)\right|^{2} d x\right) \\
&=C^{\prime \prime} \mu\left(\Omega_{k}\right)\left(\int_{\widetilde{\Omega}_{k}}\left|\nabla^{l} f(x)\right|^{2} d x+2^{-d k} \int_{\widetilde{\Omega}_{k}}|f(x)|^{2} d x\right) \\
& \leqslant 2^{d} C^{\prime \prime} \mu\left(\Omega_{k}\right)\left(\int_{\widetilde{\Omega}_{k}}\left|\nabla^{l} f(x)\right|^{2} d x+\int_{\widetilde{\Omega}_{k}} \frac{|f(x)|^{2}}{|x|^{2 l}} d x\right) .
\end{aligned}
$$

This inequality and the definition of $\mathcal{K}$ imply

$$
\int_{\bar{\Omega}_{k}}|f(x)|^{2} d \mu(x) \leqslant \frac{1}{3}\left(\int_{\widetilde{\Omega}_{k}}\left|\nabla^{l} f(x)\right|^{2} d x+\int_{\widetilde{\Omega}_{k}} \frac{|f(x)|^{2}}{|x|^{2 l}} d x\right) .
$$

Summing up the inequalities (4.3) and (4.6) we obtain (4.1). Besides, (4.4) gives $\operatorname{dim} E_{0}=\sum_{k \in \mathcal{K}} \operatorname{dim} E_{k}=\sum_{k \in \mathcal{K}} m_{k} \leqslant C(d) \mu\left(\mathbb{R}^{d}\right)$. The theorem is proved.

\section{Proof of Theorem 1.2}

5.1. Some properties of $L^{p}$ classes of functions. Let $Q=(0,1)^{d}, d \in \mathbb{N}$. We begin with an auxiliary statement.

Proposition 5.1. Let $f \geqslant 0$ and $f \in L^{p}(Q), 1<p \leqslant \infty$. Then there exists $g \in L^{p}(Q)$, such that $g \geqslant f$ a.e.,

$$
\|g\|_{L^{p}(Q)} \leqslant C(p, d)\|f\|_{L^{p}(Q)}
$$

and the measure $g d x$ satisfies Condition $(*)$ with some constants $\gamma_{1}=\gamma_{1}(p, d)$ and 
Proof. Let $u \in L^{p}\left(\mathbb{R}^{d}\right)$ and let $P u=\chi_{Q} u=\left.u\right|_{Q}$ be the restriction of $u$ to the cube $Q$. Introduce the Hardy-Littlewood maximal function

$$
\mathcal{M} f(x)=\sup _{\rho>0} \frac{1}{|B(x, \rho)|} \int_{B(x, \rho)}|f(y)| d y
$$

Then by using the Hardy-Littlewood-Wiener theorem (see for example Th.I.1 in [St]) we find that there is a constant $A=A(p)$, such that

$$
\|P \mathcal{M} f\|_{p} \leqslant\|\mathcal{M} f\|_{p} \leqslant A\|f\|_{L^{p}(Q)}
$$

Define (cf. $[\mathrm{GR}])$

$$
g(x)=\sum_{k=0}^{\infty} 2^{-k} A^{-k}(P \mathcal{M})^{k} f(x)
$$

Obviously supp $g \subset Q, f \leqslant g$ a.e., $\|g\|_{p} \leqslant 2\|f\|_{p}$, and

$$
P \mathcal{M} g(x) \leqslant 2 A g(x)
$$

It only remains to check that the measure $g d x$ satisfies Condition $(*)$. Thus we should find constants $\left(\gamma_{1}, \gamma_{2}\right)$, such that for any $x_{0} \in \mathbb{R}^{d}$ and $r \leqslant \gamma_{2}^{-1} \sqrt{d}$

$$
\int_{B\left(x_{0}, \gamma_{1} r\right)} g(x) d x \geqslant 2 \int_{B\left(x_{0}, r\right)} g(x) d x
$$

Let $\gamma_{1}=\gamma_{2}=\gamma>1$ be a constant whose value is to be found. Then for any $x \in Q \cap\left\{B\left(x_{0}, \gamma r\right) \backslash B\left(x_{0}, r\right)\right\}$ the inequality (5.2) implies

$$
\begin{aligned}
g(x) \geqslant \frac{1}{2 A\left|B\left(x, r+\left|x-x_{0}\right|\right)\right|} \int_{B\left(x, r+\left|x-x_{0}\right|\right)} g(y) d y & \\
& \geqslant \frac{1}{2 A v_{d}\left(r+\left|x-x_{0}\right|\right)^{d}} \int_{B\left(x_{0}, r\right)} g(y) d y .
\end{aligned}
$$

Integrating this inequality over the set $Q \cap\left\{B\left(x_{0}, \gamma r\right) \backslash B\left(x_{0}, r\right)\right\}$ we obtain

$$
\begin{aligned}
& \int_{Q \cap\left\{B\left(x_{0}, \gamma r\right) \backslash B\left(x_{0}, r\right)\right\}} g(x) d x \\
& \geqslant \frac{1}{2 A v_{d}} \int_{Q \cap\left\{B\left(x_{0}, \gamma r\right) \backslash B\left(x_{0}, r\right)\right\}} \frac{1}{\left(r+\left|x-x_{0}\right|\right)^{d}} d x \int_{B\left(x_{0}, r\right)} g(y) d y \\
& \geqslant \frac{1}{2^{d+1} A v_{d}} \int_{B\left(x_{0}, \gamma r\right) \backslash B\left(x_{0}, r\right)} \frac{1}{\left(r+\left|x-x_{0}\right|\right)^{d}} d x \int_{B\left(x_{0}, r\right)} g(y) d y \\
& =\frac{v_{d} d}{2^{d+1} A v_{d}} \int_{r}^{\gamma r} \frac{u^{d-1}}{(r+u)^{d}} d u \int_{B\left(x_{0}, r\right)} g(y) d y
\end{aligned}
$$

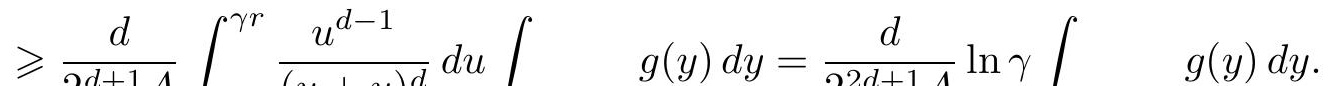


If we now choose $\gamma$, such that

$$
d \ln \gamma \geqslant 2^{2 d+2} A,
$$

then (5.3) is satisfied and therefore the proof is complete.

When defining Condition (*) we used the family of balls. There is a natural question whether instead of balls we can use other families of sets. The next lemma answers this question.

Let $0 \in G \subset \mathbb{R}^{d}$ be a domain, such that

$$
B\left(0, r_{1}\right) \subset G \subset B\left(0, r_{2}\right)
$$

with some $0<r_{1}<r_{2}<\infty$. Define

$$
G(x, r)=\left\{y \in \mathbb{R}^{d}:(y-x) / r \in G,\right\}, \quad r>0 .
$$

Lemma 5.2. Let $G$ be a set satisfying (5.4) and let $\mu$ be a non-negative measure, $F=\operatorname{supp} \mu$. The following two properties are equivalent:

(i) There exist constants $\gamma_{1}$ and $\gamma_{2}$, such that Condition (*) holds with constants $\left(\gamma_{1}, \gamma_{2}\right)$

(ii) There exist constants $\gamma_{1}^{\prime}$ and $\gamma_{2}^{\prime}$, such that for any $x \in \mathbb{R}^{d}$ and $r \leqslant \operatorname{diam} F / \gamma_{2}^{\prime}$ we have

$$
\mu\left(G\left(x, \gamma_{1}^{\prime} r\right)\right) \geqslant 2 \mu(G(x, r)) .
$$

Proof. Suppose (i) is satisfied. Let us check (ii). For any $x \in \operatorname{supp} \mathbb{R}^{d}$ and $r \leqslant \frac{\operatorname{diam} F}{\gamma_{2} r_{2}}$ we have that

$$
\mu\left(G\left(x, \gamma_{1} r_{2} r / r_{1}\right)\right) \geqslant \mu\left(B\left(x, g_{1} r_{2} r\right) \geqslant 2 \mu\left(B\left(x, r_{2} r\right)\right) \geqslant 2 \mu(G(x, r)) .\right.
$$

The latter implies $\gamma_{1}^{\prime}=\gamma_{1} r_{2} / r_{1}$ and $\gamma_{2}^{\prime}=r_{2} \gamma_{2}$. The converse statement can be proved analogously.

In the proof of the next statement it is convenient to use a family of cubes

$$
\begin{gathered}
Q(x, r)=\left\{y \in \mathbb{R}^{d}:|y-x| / r \in(-1,1)^{d}\right\}, \\
Q_{1}\left(x_{1}, r\right)=\left\{y_{1} \in \mathbb{R}^{d_{1}}:\left|y_{1}-x_{1}\right| / r \in(-1,1)^{d_{1}}\right\}, \\
Q_{2}\left(x_{2}, r\right)=\left\{y_{2} \in \mathbb{R}^{d_{2}}:\left|y_{2}-x_{2}\right| / r \in(-1,1)^{d_{2}}\right\} .
\end{gathered}
$$

Proposition 5.3. Let $Q=Q_{1} \times Q_{2}=(0,1)^{d_{1}} \times(0,1)^{d_{2}}, d=d_{1}+d_{2}, f \geqslant 0$ and $f \in L^{1}\left(Q_{1}, L^{p}\left(Q_{2}\right)\right), 1<p \leqslant \infty$. Then there exists $g \in L^{1}\left(Q_{1}, L^{p}\left(Q_{2}\right)\right)$, such that $g \geqslant f$ a.e.,

$$
\|g\|_{L^{1}\left(Q_{1}, L^{p}\left(Q_{2}\right)\right)} \leqslant C\left(p, d_{1}, d_{2}\right)\|f\|_{L^{1}\left(Q_{1}, L^{p}\left(Q_{2}\right)\right)}
$$

and the measure $g d x$ satisfies Condition $(*)$ with the constants $\gamma_{1}=\gamma_{1}\left(p, d_{1}, d_{2}\right)$ and $\gamma_{2}=\gamma_{2}\left(p, d_{1}, d_{2}\right)$.

Proof. For the functions $f\left(x_{1}, \cdot\right) \in L^{p}\left(Q_{2}\right), x_{1} \in Q_{1}$, we introduce $g\left(x_{1}, \cdot\right) \in L^{p}\left(Q_{2}\right)$ according the construction in Proposition 5.1. Clearly $g\left(x_{1}, x_{2}\right) d x_{2}$ satisfies Condition $(*)$ for the family of cubes $Q_{2}\left(x_{2}, r\right), x_{2} \in \mathbb{R}^{d_{2}}$, with constants $\left(\gamma_{1}, \gamma_{2}\right)$ uni- 
we prove (5.6) for the family of cubes $Q(x, r), x=\left(x_{1}, x_{2}\right)$. Indeed, for any $x \in \mathbb{R}^{d}$ and $r<\sqrt{d} / \gamma_{2}$ we have

$$
\begin{aligned}
\int_{Q\left(x, \gamma_{1} r\right)} g(y) d y= & \int_{Q_{1}\left(x, \gamma_{1} r\right)} \int_{Q_{2}\left(x, \gamma_{1} r\right)} g\left(y_{1}, y_{2}\right) d y_{2} d y_{1} \\
& \geqslant 2 \int_{Q_{1}\left(x, \gamma_{1} r\right)} \int_{Q_{2}(x, r)} g\left(y_{1}, y_{2}\right) d y_{2} d y_{1} \\
& \geqslant 2 \int_{Q_{1}(x, r)} \int_{Q_{2}(x, r)} g\left(y_{1}, y_{2}\right) d y_{2} d y_{1} .
\end{aligned}
$$

The proposition is proved.

Corollary 5.4. The statement of Proposition 5.3 holds true if we replace the cube $Q_{2}$ by $\mathbb{S}^{d_{2}}$.

5.2. Proof of Theorem 1.2. In the polar coordinates $x=(r, \theta) \in \mathbb{R}^{+} \times$ $\mathbb{S}^{d-1}$, every set $\Omega_{k}$ turns into $\left[2^{k}, 2^{k+1}\right) \times \mathbb{S}^{d-1}$. According to Proposition 5.3 we find functions $g_{k} \in L^{1}\left(\left(2^{k}, 2^{k+1}\right), L^{p}\left(\mathbb{S}^{d-1}\right)\right)$, such that $g:=\sum_{k} g_{k} \geqslant V$ a.e., $\|g\|_{L^{1}\left(\mathbb{R}_{+}, L^{p}\left(\mathbb{S}^{d-1}\right)\right)} \leqslant C(p, d)\|V\|_{L^{1}\left(\mathbb{R}_{+}, L^{p}\left(\mathbb{S}^{d-1}\right)\right)}$ and the measures $g_{k} d x$ satisfy Condition $(*)$ with constants $\left(\gamma_{1}, \gamma_{2}\right)$ which are independent of $k$. Finally we have

$$
N(V) \leqslant N(g) \leqslant C_{1} \int g(x) d x \leqslant C_{2}\|g\|_{L^{1}\left(\mathbb{R}_{+}, L^{p}\left(\mathbb{S}^{d-1}\right)\right)} \leqslant C_{3}\|V\|_{L^{1}\left(\mathbb{R}_{+}, L^{p}\left(\mathbb{S}^{d-1}\right)\right)}
$$

where $C_{j}=C_{j}(d, p), j=1,2,3$. This completes the proof.

\section{REFERENCES}

[A] D.R. Adams, Traces of potentials arising from translation invariant operators, Ann. Scuola Norm. Sup. Pisa Cl. Sci. 25 (1971), 203-217.

[AH] D.R. Adams and L.I. Hedberg, Function Spaces and Potential theory, Springer, 1996.

[B1] A.S. Besicovitch, A general form of the covering principle and relative differentiation of additive functions, Proc. Cambridge Philos. Soc. 41 (1945), 103-110.

[B2] A.S. Besicovitch, A general form of the covering principle and relative differentiation of additive functions, Proc. Cambridge Philos. Soc. 42 (1946), 1-10.

[BL] M.Sh.Birman and A.Laptev, The negative discrete spectrum of a two-dimensional Schrödinger operator, Comm. Pure Appl. Math. XLIX (1996), 967-997.

[BLS] M.Sh. Birman, A. Laptev and M. Solomyak, The negative discrete spectrum of the operator $(-\Delta)^{l}-\alpha V$ in $L_{2}\left(\mathbb{R}^{d}\right)$ for $d$ even and $2 l \geqslant d$, Ark. Mat. 35 (1997), 87-126.

[BS1] M.Sh.Birman and M.Z.Solomyak, Estimates for the number of negative eigenvalues of the Schrödinger operator and its generalizations., Advances in Soviet Math. 7 (1991), $1-55$.

[BS2] M.Sh.Birman and M.Z.Solomyak, Quantitive analysis in Sobolev imbedding theorems and applications to spectral theory., Tenth Mathem. School., Izd. Inst. Mat. Akad. Nauk Ukrain, SSSR, Kiev, 1974, pp. 5-189 (Russian); English transl. in Amer. Math. Soc. Transl. (2), vol. 114, 1980, pp. 1-132.

[C] H. Cartan, Sur les systèmes de fonctions holomorphes à variétés linéaires et leurs applications, Ann. École Norm. Sup. 3 (1928), 255-346.

[GR] J. García-Cuerva and J.L. Rubio de Francia, Weighted Norm Inequalities and Related 
[G] M. de Guzmán, Differentiation of integrals in $\mathbb{R}^{n}$, vol. 481, Lecture Notes in Mathematics, 1975.

[L] A. Laptev, On the negative spectrum of a class of Schrödinger operators with spherically symmetric potentials, preprint KTH, Sweden. (1998).

[M] V. Maz'ya, Sobolev Spaces, Springer-Verlag, Berlin, Heidelberg, New York, Tokyo, 1985.

[R] G.V.Rosenblum, Distribution of the discrete spectrum of singular differential operators., Izv. Vyssh. Uchebn. Zaved. Mat. 1 (1976), 75-86 (Russian); English transl. in Soviet Math. Izv. VUZ 20 (1976), 63-71.

[S1] M.Solomyak, Piecewise-polynomial approximation of functions from $H^{l}\left((0,1)^{d}\right), 2 l=d$, and applications to the spectral theory of Schrödinger operator, Israel J. Math. 86 (1994), 253-276.

[S2] M.Solomyak, Spectral problems related to the critical exponent in the Sobolev embedding theorem, Proc. London Math. Soc. 71 (1995), 53-75.

[St] E.M. Stein, Singular Integrals and Differentiability of Functions, Princeton University Press, Princeton, New Jersey, 1970.

Department of Mathematics, Royal Institute of TEChNOLOGy, S-100 44 Stockholm, Sweden

E-mail address: laptev@math.kth.se

Department of Mathematics, King's College, Strand, LONDON WC2R 2LS, ENGLAND AND

POMI, Fontanka 27, St.Petersburg, 191011 RUSSIA

E-mail address: netrusov@mth.kcl.ac.uk 